\title{
Review of: "Asperosaponin VI Ameliorates The CMS- Induced Depressive-Like Behaviors By Inducing A Neuroprotective Microglial Phenotype To Restore Hippocampal Synaptic Plasticity Via PPAR-y Pathway"
}

\author{
Ming Zhang
}

Potential competing interests: The author(s) declared that no potential competing interests exist.

This manuscript from Dr. Jinqiang Zhang's group investigated the molecular mechanisms for how Asperosaponin VI (ASA VI), which is one of active components of the traditional Chinese medicine Radix Dipsaci, ameliorates chronic mild stress (CMS)-induced depression-like behaviors in mice. Of course it is important to explore novel antidepressants and the underlying mechanisms for the treatment of Major depressive disorder (MDD). In the study, the authors demontrate that ASA VI treatment effectively reversed CMS-induced pro-inflammatory microglial phenotype, while promoted neuroprotective microglial phenotype, in a PPAR- $\gamma$-dependent manner, to exert its antidepressant effect on stressful animals. ASA VI also effectively reversed CMS-induced abnormal decrease of neuron-microglia communication molecules and synaptic function relevant proteins. Overall, the authors have shown in the manuscript that ASA VI is a potential natural antidepressant through exerting its beneficial effects on hippocampal microglia and neuronal functions.

Some concerns to be addressed are listed in the following.

(1) In the "Introduction", some general background knowledge about PPAR- $\gamma$ and the reason that the authors intend to examine the potential involvement of the PPAR-y pathway in mediating the effects of asperosaponin VI should be given.

(2) In the "Materials And Methods", there are some questions:

I. The animal strain should keep consistent.In "2.1 Animals", the animal strain is C57BL/6 mice, but in Figure $1 \mathrm{~A}$, it is $\mathrm{C} 57 \mathrm{BL} / 6 \mathrm{~J}$.

II. If it is true that "All mice were housed individually", is it possible that the individually housing led to a situation of "social isolation" which could affect the emotional output in these mice?

III. Why did the experimenters perform the FST for two days, and record the time spent immobile on the second day? The immobility time recorded on the first day is widely employed in FST to analyze depresson-like behaviors.

IV. In 2.6 Immunocytochemistry, 2.7 ELISA, and 2.8 Western blotting, detailed information about the most of primary antibodies and secondary antibodies are missing. 
(3) As shown in Figure $1 \mathrm{~A}$ and Figure 4C, did the experimenters perform behavioral testings of OFT,NSFT,TST,FST on the only one day (43d)? Whether it is true or not, please add some sentences to clarify the order of behavioral testing and the specific day and time during the whole testing procedure.

(4) How did the experimenters treat the control animal should be clarified in the "Materials And Methods". And in Figure 1, please notice that the meanings of the "Ctrl" group and the "CMS" group are not equal to the "Ctrl+saline" group and the "CMS+saline" group.

(5) The authors found that ASA VI did not alter the CMS-induced radial morphology of astrocytes in the hippocampus and cortex (Fig. 2D-2F). The figures of 2D-2F are not just the examination of morphology of astrocytes, please state these results completely, and add the biological meaning of these results of astrocytes.

(6) To draw a conclusion that ASA VI treatment can restore hippocampal synaptic plasticity in CMSexposed mice, it is not enough to just show the expression of PSD95, CaMKII $\alpha$, CaMKII $\beta$ and p-GluA2 in hippocampus since many molecules besides these four proteins are involved in the regulation of synaptic plasticity. If the authors intend to keep this conclusion, then electrophysiological data (at least for LTP) are prefered.

(7) By the way, as shown in Fig.6C and 6D, ASA VI significantly reversed the decrease of PSD95, CaMKIl $\alpha$, CaMKII $\beta$ and p-GluA2 in hippocampus when compared to the CMS only group, and the averaged expression levels of these proteins after ASA VI treatment were even higher when compared to the control group, so it was not just "partially reversed by asperosaponin VI" as stated at the page 7 and the page 9 .

(7) In the Discussion, the author's previous work ([23] Zhang J, Yi S, Li Y, Xiao C, Liu C, Jiang W, Yang C, Zhou T: The antidepressant effects of asperosaponin VI are mediated by the suppression of microglial activation and reduction of TLR4/NF-kappaB-induced IDO expression. Psychopharmacology (Berl) 2020) found asperosaponin VI effectively reversed depression-like behaviors. The comparison between the two studies should provide a clearer sight on how the compound exert its antidepressant effects, at least including why ASA VI inhibited LPS-induced activation of microglia in the previous study vs ASA VI did not show obvious reversal effect on CMS-induced microglia activation in the present study, and the speculated reason that the same compound (i.e. ASA VI) affects different signaling pathways (TLR4/NF-kappaB-IDO vs PPAR- $\gamma$ ) between the two studies.

(8) Questions about references:

I. Please add original reference(s) for the statement that $\mathrm{Cd} 1 \mathrm{lb}$ is the marker of microglial activation, 
and the pairs of CX3CL1/CX3CR1 and CD200/CD200R are the markers of interactions between hippocampal microglia and neurons.

II. At page 8 , it is stated that "Our previous research indicated that the dysregulation of pro- and antiinflammatory cytokines plays a crucial role in depression [8]. "You Z, Luo C, Zhang W, Chen Y, He J, Zhao Q, Zuo R, Wu Y: Pro- and anti-inflammatory cytokines expression in rat's brain and spleen exposed to chronic mild stress: involvement in depression. Behav Brain Res 2011, 225:135-141.", but this reference does not match any author in the present study.

III.At page 9, it is stated that "the PPAR-y agonists pioglitazone or rosiglitazone can switch activated microglia cells from a pro-inflammatory to anti-inflammatory state [36,37], but I can not find the data of pioglitazone in these two references. The authors should check references thoroughly for apprepriate.

"36. Ji J, Xue TF, Guo XD, Yang J, Guo RB, Wang J, Huang JY, Zhao XJ, Sun XL: Antagonizing peroxisome proliferator-activated receptor $\mathrm{Y}$ facilitates M1-to-M2 shift of microglia by enhancing autophagy via the LKB1-AMPK signaling pathway. Aging Cell 2018, 17:e12774.

37. Peng J, Wang K, Xiang W, Li Y, Hao Y, Guan Y: Rosiglitazone polarizes microglia and protects against pilocarpine-induced status epilepticus. CNS Neurosci Ther 2019, 25:1363-1372." 